\title{
Smart Colloid-Assisted Technique Prompts the Evolution of Bamboo Wastes into Nanometals-Inlaid Carbon Microfibers for Sustainable Ni-Fe Batteries
}

Lai $\mathrm{Ma}^{\mathrm{a}}$, Yihan $\mathrm{Xu}^{\mathrm{c}}$, Yani $\mathrm{Liu}^{\mathrm{a}}$, Han Zhanga ${ }^{\mathrm{a}}$, Jiajia $\mathrm{Yao}^{\mathrm{b}}$, Ning $\mathrm{Li}^{\mathrm{a}}$, Chang Ming $\mathrm{Li}^{\mathrm{a}}$,* Weiwei Zhou $^{\mathrm{c}, *}$ and Jian Jiang,*

${ }^{a}$ School of Materials and Energy, and Chongqing Key Lab for Advanced Materials and Clean Energies of Technologies, Southwest University, No. 2 Tiansheng Road, BeiBei District, Chongqing 400715, P.R. China

${ }^{b}$ School of Physical Science and Technology, Southwest University, No.2 Tiansheng Road, BeiBei District, Chongqing 400715, P.R. China.

'School of Materials Science and Engineering, Harbin Institute of Technology at Weihai, No.2 Wenhua Road, Weihai 264209, P.R. China.

To whom correspondence should be addressed: Tel: +86-23-68254842.

"E-mail: zhouweiwei@hit.edu.cn (W.W.Zhou); jjiang@swu.edu.cn (J. Jiang); ecmli@swu.edu.cn (C.M.Li).

\section{Supporting Information for Publication available:}

On page S1: Cover sheet;

On Page S2: Figure S1: XRD detection toward the intermediate colloidal substance.

Figure S2: Typical cross sectional SEM observations of Fe@CMFs fibrous products.

On Page S3: Figure S3: Typical Raman spectra of bare CMFs and Fe@CMFs.

On Page S4: Figure S4: TGA analysis toward Fe@CMFs and Ni@CMFs hybrids in air atmospheres.

On Page S5: Figure S5: Optical images of BCMFs soaked in salt-involved (a) absolute ethanol and (b) water solvent.

On Page S6: Figure S6: FT-IR spectra of BCMFs before soaking (red) and after soaking (black) in salt-involved absolute ethanol.

Figure S7: XRD patterns of Fe@CMFs at different temperatures.

On Page S7: Figure S8: SEM observations of Fe@CMFs under different temperature conditions.

Figure S9: Typical SEM observations on cycled electrodes: (a-b) Fe@CMFs; (c-d) Ni@CMFs. 


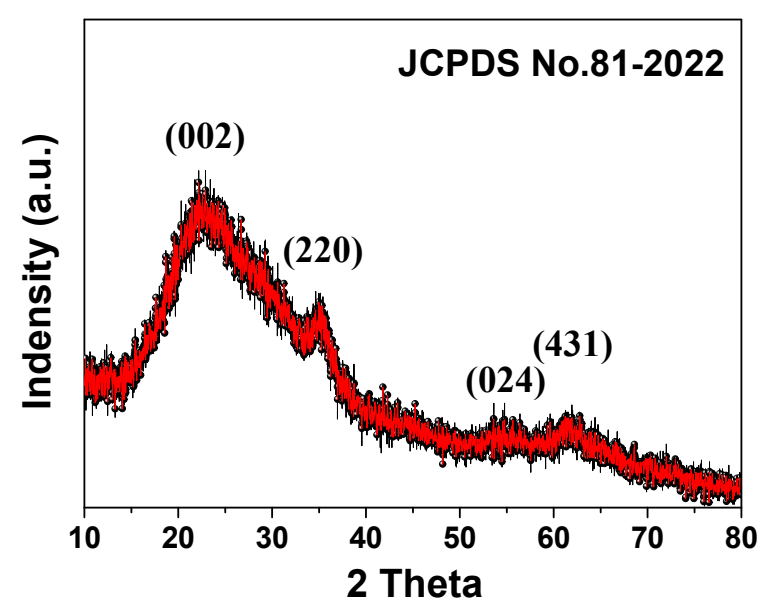

Figure S1. XRD detection toward the intermediate colloidal substance.
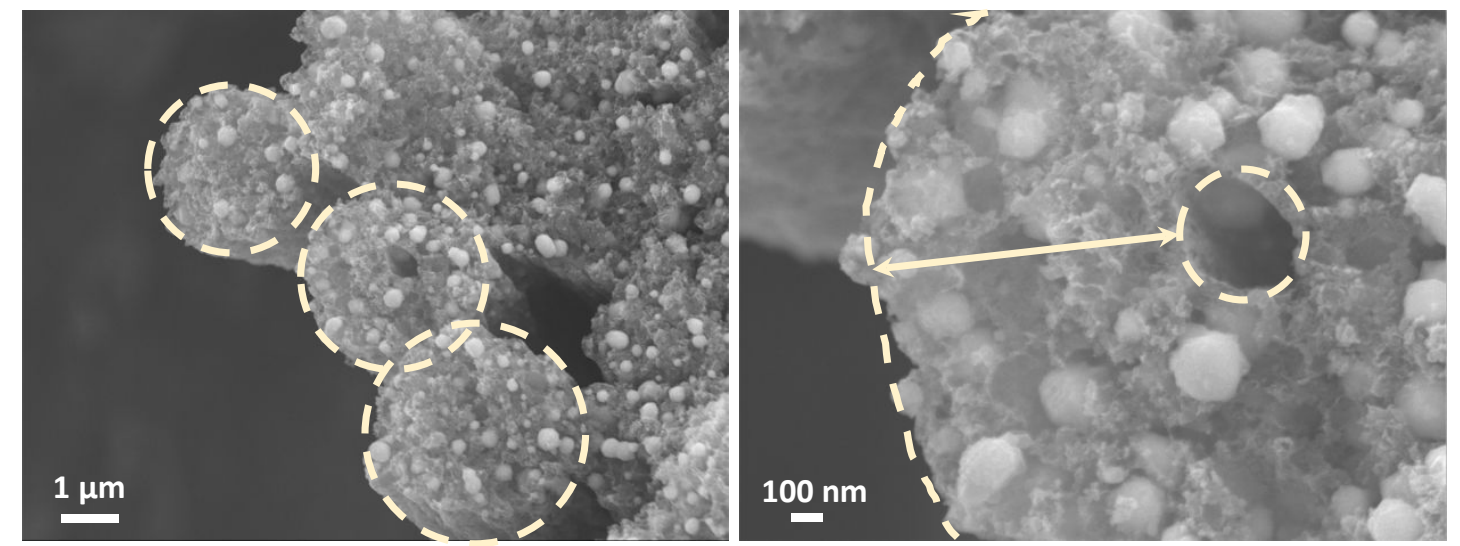

Figure S2. Typical cross sectional SEM observations of Fe@CMFs fibrous products. 


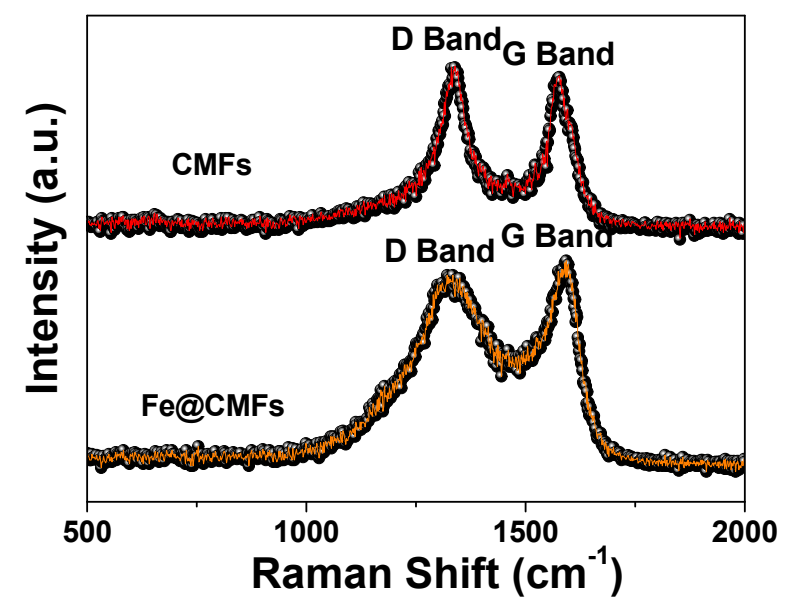

Figure S3. Typical Raman spectra of bare CMFs and Fe@CMFs. 

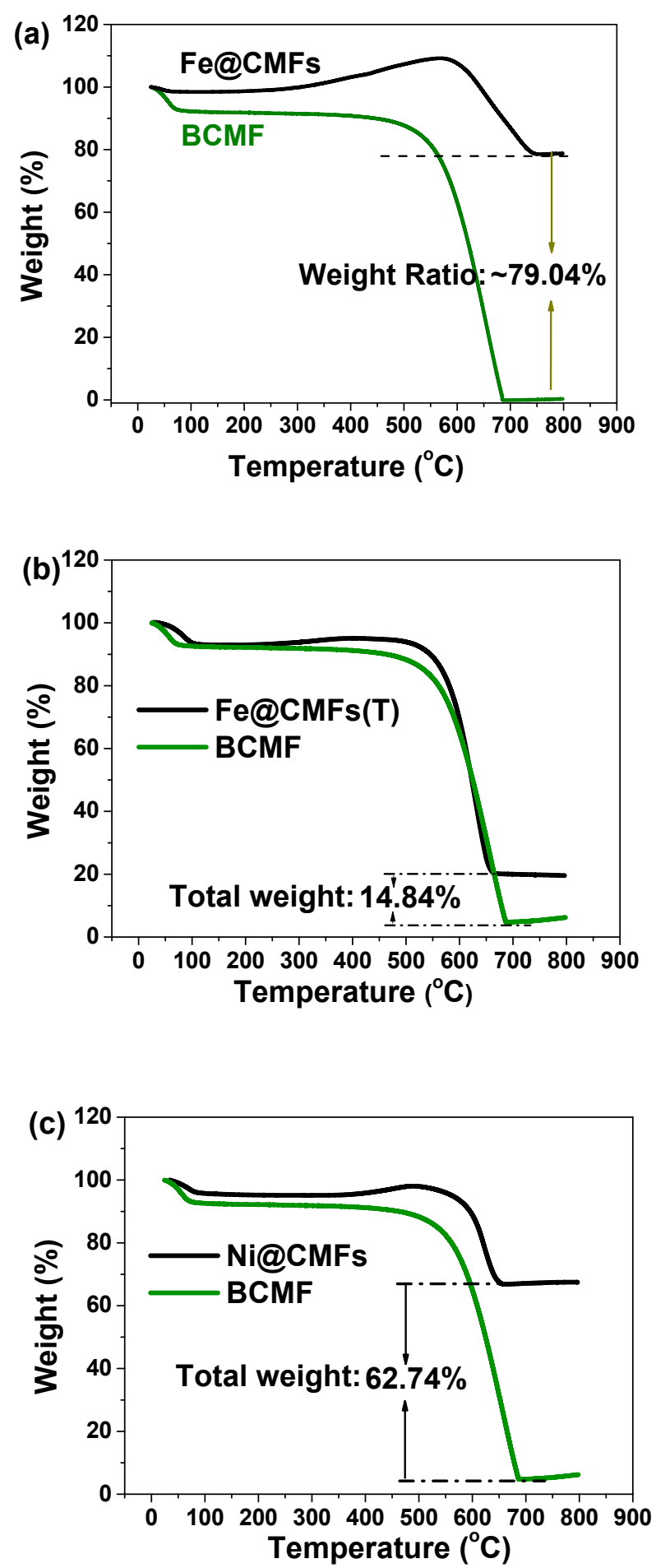

Figure S4. TGA analysis toward (a) Fe@CMFs, (b) Fe@CMFs (T) and (c) Ni@CMFs hybrid samples in air atmospheres. 


\section{Composition calculation:}

The total $\mathrm{C}$ content in samples can be estimated by the reaction equation for $\mathrm{Fe}$ in air atmospheres: $4 \mathrm{Fe}+3 \mathrm{O}_{2} \rightarrow 2 \mathrm{Fe}_{2} \mathrm{O}_{3}$. According to the mass for yielded $\mathrm{Fe}_{2} \mathrm{O}_{3}$ and molar ratio relationships in between $\mathrm{Fe}$ and $\mathrm{Fe}_{2} \mathrm{O}_{3}$, we can eventually determine the Fe content in $\mathrm{Fe} @$ CMFs hybrids. By referring to TGA data, the mass ratio for metallic $\mathrm{Fe}$ is calculated to be $\sim 55.3 \%(\sim 79.04 \% / 160) \times 2 \times$ $56=\sim 55.3 \%$ ). Thus, the mass ratio of $\mathrm{C}$ in hybrids can be estimated at the level of $\sim 44.7 \%$ $(1-55.3 \%=\sim 44.7 \%)$. Besides, the total Ni content in Ni@CMFs can be also determined by the reaction equation of $2 \mathrm{Ni}+\mathrm{O}_{2} \rightarrow 2 \mathrm{NiO}$. The mass ratio for metallic $\mathrm{Ni}$ is eventually calculated to be $\sim 49.36 \%((62.74 \% / 75) \times 1 \times 59=\sim 49.36 \%)$.

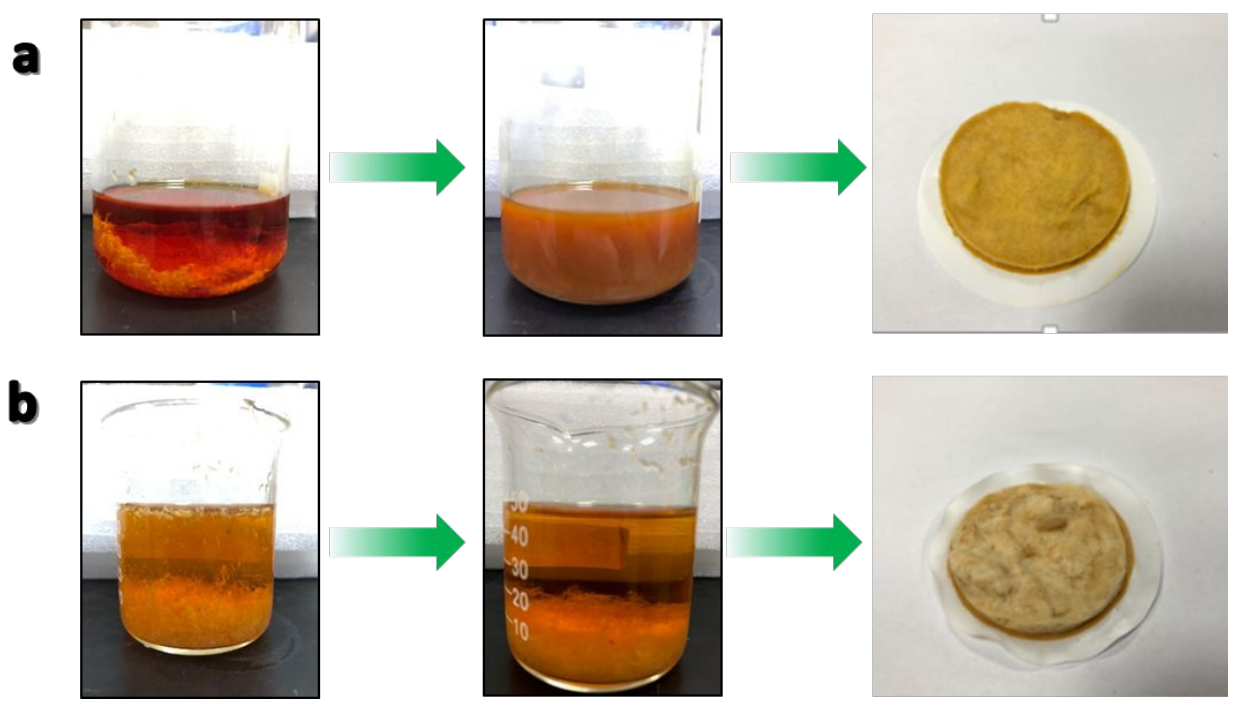

Figure S5. Optical images of BCMFs soaked in salt-involved (a) absolute ethanol and (b) water solvent. 


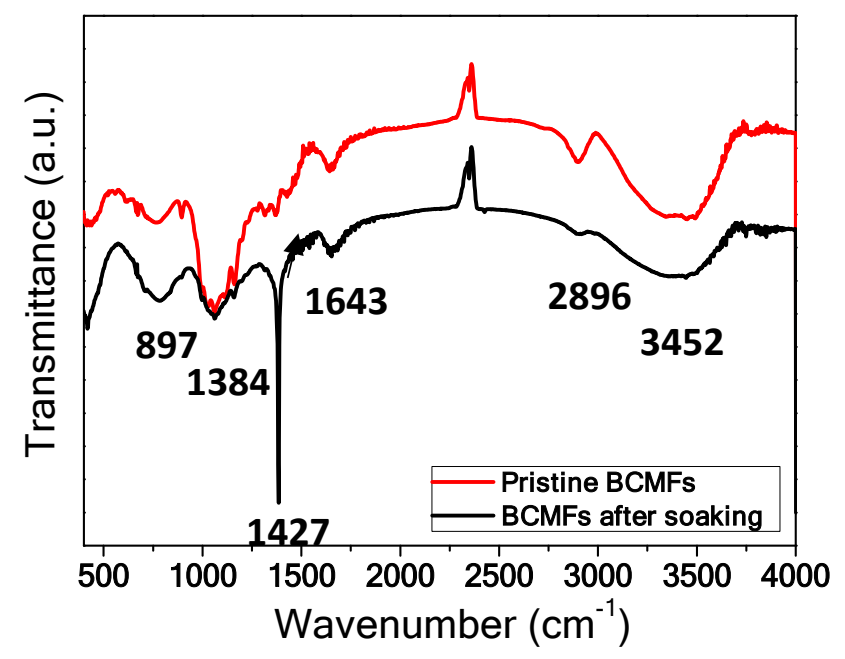

Figure S6. FT-IR spectra of BCMFs before soaking (red) and after soaking (black) in salt-involved absolute ethanol.

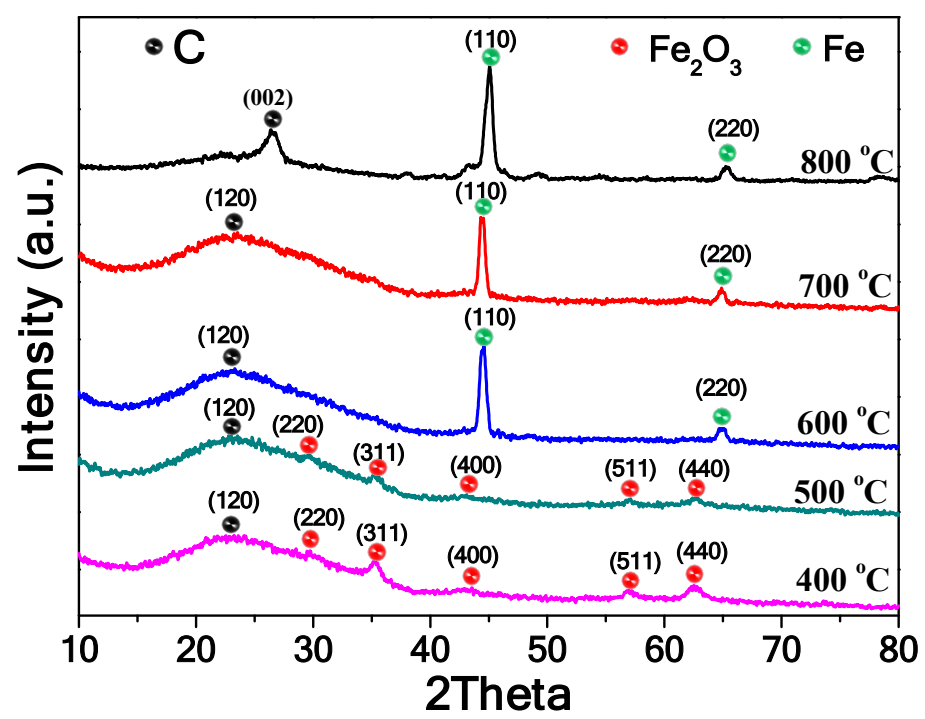

Figure S7. XRD patterns of Fe@CMFs at different temperatures. 


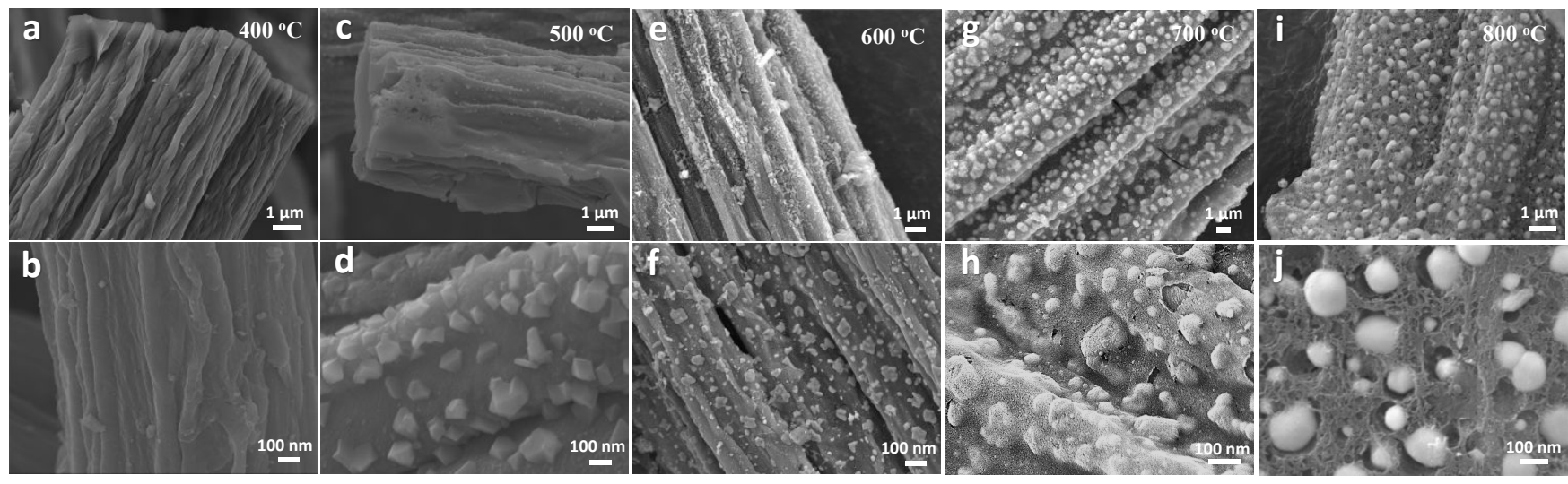

Figure S8. SEM observations of Fe@CMFs under different temperature conditions.
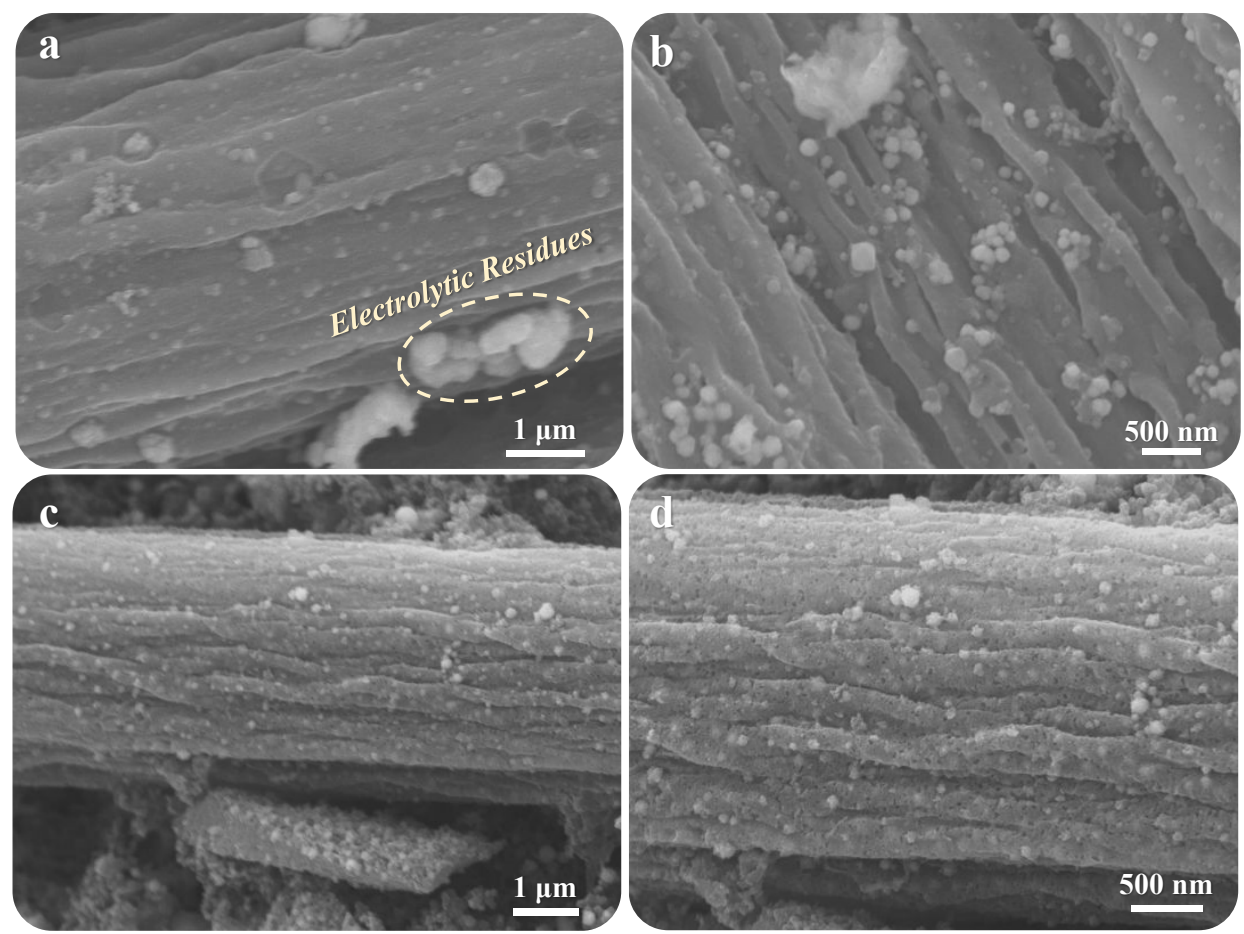

Figure S9. Typical SEM observations on cycled electrodes: (a-b) Fe@CMFs; (c-d) Ni@CMFs. 